\title{
Über das Verhältnis des Französischen zu den einheimischen Sprachen im postkolonialen Afrika: Eine Bestandsaufnahme
}

\author{
Lacina Yéo \\ Université de Cocody-Abidjan, 08 BP 724 Abidjan 08, Côte d'Ivoire \\ Freie Universität Berlin, Habelschwerdter Allee 45, 14195 Berlin-Dahlem \\ E-mail: tandoy2004@yahoo.fr
}

\begin{abstract}
Early intercultural contact occurred in West Africa through transatlantic slavery and colonisation. This led to the development of hybridized cultures in many parts of the world. Depending on the historical context in different communities, this cultural hybridization has manifested itelf in linguistic forms and is currently central in post-colonical discourse. It is possible to trace current power relations and social hierarchies in a particular community back to the relationship between colonial languages (which in many cases are used as official languages, languages of business and languages in education) and the indigenous local languages (which were disregarded in colonial times). This paper considers how the relationship between French and indigenous African languages, in such a context, is to be estimated in various domains of use.
\end{abstract}

Keywords: colonial languages, African languages, postcolonialism, linguistic and cultural hybridity Schlüsselbegriffe: Kolonialsprachen, afrikanische Sprachen, Postkolonialismus, sprachliche und kulturelle Hybridität

\section{Einleitung}

Die Assimilationspolitik Frankreichs, die sich durch eine direkte Verwaltung der Kolonien, die sprachliche, religiöse und kulturelle Assimilation ihrer Einwohner und die wirtschaftliche Ausbeutung der Kolonialgebiete kennzeichnete, hat die Förderung der lokalen afrikanischen Sprachen stark beeinträchtigt. In der Kolonialzeit wurden z.B. SchülerInnen bestraft, die ihre Muttersprache in der Klasse oder auf dem Schulhof sprachen. Französisch fungiert somit als kulturpolitisches Unterdrückungsmittel, daher die gegenwärtige Kritik am neokolonialen Charakter der Frankophonie (vgl. Fall 1996:3).

Nach der Unabhängigkeit wurde Französisch in ganz Subsahara-Afrika als Verkehrs-, Amts-, Unterrichts- und Literatursprache beibehalten. Wie aus einer Untersuchung zum Hilfsprogramm der UNESCO für den Aufbau staatlicher Schulsysteme in Subsahara-Afrika (Kirschbaum 1995) hervorgeht, unternahm die UNESCO in den 60er Jahren den Versuch, als Unterrichtssprache afrikanische Sprachen einzusetzen. Jedoch stieß die Organisation auf den Widerstand Frankreichs. So hatte die UNESCO kaum Möglichkeiten, in den französischen Kolonien Studien über die Bevölkerung und deren tatsächlichen bildungspolitischen 
Bedürfnisse und Voraussetzungen $\mathrm{zu}$ erstellen oder Projekte der Grunderziehung durchzuführen (vgl. Yéo 2003:329f).

Doch trotz der Vorrangstellung der französischen Sprache im postkolonialen Afrika werden afrikanische Idiome im alltäglichen Leben als Kommunikationssprachen weiter verwendet. Die Feststellung ist sogar gerechtfertigt, dass die lokalen afrikanischen Sprachen dem Französischen sowohl in der Umgangs- als auch in der Literatursprache jeweils in Form einer „Nouchi““1 - oder Kreolsprache und von bikulturellen oder hybriden Texturen ihren Stempel aufdrücken. Die vorliegende Untersuchung ist allen Formen von Interferenzen zwischen dem Französischen und den einheimischen afrikanischen Sprachen sowohl im alläglichen Leben als auch im Literaturbetrieb gewidmet.

\section{Die intellektuelle Auseinandersetzung mit dem kolonialkulturellen Erbe am Beispiel der französischen Sprache}

Seit den sechziger Jahren findet in Afrika eine intellektuelle Auseinandersetzung mit dem kulturellen Erbe der europäischen Kolonisation statt. Dabei gibt es sowohl Befürworter als auch Gegner der Beibehaltung der europäischen Sprachen als Verkehrs-, Amts-, Unterrichtsund Literatursprache im postkolonialen Afrika. Im frankophonen Afrika war der senegalesische Politiker und Dichter Léopold Sédar Senghor einer der bekanntesten Verfechter der französischen Sprache. Für ihn wie für viele andere Intellektuelle in seiner Nachfolge könne die französische Sprache den Afrikanern den Anschluss an die moderne Welt ermöglichen:

La langue française constitue, pour nous, une langue de communication entre nos États et les différents groupes ethniques à l'intérieur du même États; elle est aussi la langue qui nous soude au reste du monde. De par la finesse et la force de l'écriture, elle a accumulé des vertus en traversant les siècles; ces vertus peuvent exercer une influence heureuse sur le développement moderne de nos propres langues, qui commencent à se soumettre aux exigences de l'écriture. Nos langues ont aussi leurs vertus qui sont celles de l'oralité; elles sont donc capables de rendre la langue française plus dynamique et plus colorée à travers ses nuances. Témoin nos œuvres majeures, comme celles de Léopold Sédar Senghor et d'Aimé Césaire. (Gassama 1995:13)²

Die folgenden Erörterungen, die einer relativ neuen Studie zu entnehmen sind, zeugen von einem Fortwirken der obigen Denkansätze: „die englische und französische Sprache [vereinfachen] die Verständigung zwischen Afrikanern einerseits und zwischen Afrikanern und Europäern andererseits“ (Kita 2008:78). Der Kameruner Sprachwissenschaftler Gervais Mendo Ze fordert auf, Französisch nicht mehr als verlängertes Instrument (neo)kolonialer Machtverhältnisse zu begreifen, sondern es als eine Sprache zu betrachten ,qui, aujourd'hui, a pris corps dans nos vies, et qui peut exprimer notre identité culturelle et permettre de cerner ce que nous partageons avec plusieurs peuples dans le monde“ (Mendo Ze 1999:27). ${ }^{3}$

Auch der kamerunische Schriftsteller J. P. Makouta-Mboukou (1973) fordert die Afrikaner auf, sich die französische Sprache anzueignen:

Il ne faut pas que les Négro-africains subissent simplement une langue qui leur est totalement étrangère, il faut qu'ils ne soient plus de simples et mauvais consommateurs de langue française, 
mais qu'ils la recréent pour la rendre accessible à leur mode de vie et à leur manière de penser. (Zitiert nach Erfurt 2005:51)

Doch den obigen Denkansätzen stehen die folgenden Gedanken gegenüber:

Französisch ist die Sprache der Franzosen, und repräsentiert die französische Kultur. Das Abstraktum, das sie beschreibt, die Assoziationen, die bestimmte Wörter oder Redewendungen hervorrufen, kurz, die Realität, die sie mitteilt, sind im französischen kulturellen Kontext zu verstehen. (Tene 2004:9)

Im Unterschied zu Senghor ist z.B. der kamerunische Schriftsteller Mongo Beti der Meinung, dass Französisch eine Sprache der Unterdrückung und der Ausbeutung der Afrikaner darstellt; sie hätte keine Zukunft in Afrika und würde nur wenige Jahrzehnte Senghor überleben (Erfurt 2005:48ff). Zu den frankophoniekritischen Autoren gehört auch der aus Benin stammende Literaturwissenschaftler und Essayist Guy Ossito Midiohouan. Im Anschluss an die Aufnahme Senghors 1983 in die Académie francaise, reagierte er in der von Mongo Beti herausgegebenen Zeitschrift Peuples noirs, peuples africains [Schwarze Völker, afrikanische Völker] mit dem Pamphlet „La gloire du collabo“5 (vgl. Erfurt 2005:115).

\section{Einige Zahlen und Fakten zur Wahrnehmung der Entwicklungsdynamik der französischen Sprache in Afrika}

Französisch wird heute in 18 Staaten Afrikas als offizielle Sprache und als Verkehrs-, Amts-, Unterrichts-, Literatur- und Mediensprache verwendet. Dennoch gibt es Hinweise darauf, dass „weniger als 10\% der Afrikaner [Französisch] verstehen, weniger als 1\% [es] sprechen, einer von Tausend auf französisch [denkt]“.6 Folgende Angaben dienen einer detaillierten und präziseren Situationsschilderung. Sie zeigen, wie Französisch bislang nur zu einem geringen Prozentsatz Erstsprache für Afrikaner ist:

Nur ein kleiner Teil der Afrikaner hat Zugang zum Französischen. Je nach Land oder Region schwankt die Zahl der realen Frankophonen zwischen 0,1 und optimistischen 35 Prozent, meist wohl um die fünf bis zehn Prozent, jene der okkasionellen Frankophonen zwischen vier und wiederum sehr optimistischen 40 Prozent (vgl. AFI 1998ff.). Aus diesen Angaben geht nicht hervor, wie viele der einstigen Schüler, die Französisch in der Schule gelernt haben, diese Sprache schon bald wieder vergessen haben. (Erfurt 2005:49)

Es wird darüber berichtet, dass die afrikanische Literatur in den kolonialen Fremdsprachen Schwierigkeit habe, ,auch in Afrika ein Lesepublikum zu finden“, weil „weniger als 20\% der ehemals kolonisierten Bevölkerungen des sogenannten frankophonen Afrika die koloniale Sprache lesen können und sich noch weniger für die Literatur in dieser Sprache interessieren“ (Fall 1996:199). Bemerkenswert ist außerdem, dass die Entwicklung des Französischen einen Statuswandel als offizielle Sprache aufweist. In Ländern wie Ruanda, Madagaskar, Mauritius und der Demokratischen Republik Kongo, gewinnt die Verwendung des Englischen peu à peu die Oberhand über das Französische (vgl. a.a.O.:188f). 


\section{Formen von Interferenzen zwischen dem Französischen und den lokalen afrikanischen Sprachen im alltäglichen Sprachgebrauch und in der Literatur}

Eine der ausgeprägten Ausdrucksformen der Interferenzen zwischen der französischen Sprache und den einheimischen afrikanischen Sprachen besteht aus der Afrikanisierung der französischen Sprache. Zum Beispiel wird mit dem Nouchi an der Côte d'Ivoire ein afrikanisches Französisch u.a. in der Öffentlichkeit, besonders auf den afrikanischen Märkten, gesprochen, nämlich das so genannte français de Moussa oder petit nègre, das sich durch verschiedene Variationen auszeichnet.

Durch die Afrikanisierung der auf Französisch geschriebenen literarischen Texte, versuchen manche afrikanische Schriftsteller, das Besondere ihrer Kultur mit den Mitteln einer Fremdsprache zu vermitteln. Die Afrikanisierung der französischen Sprache schlägt sich nämlich auch in bedeutenden Werken der afrikanischen Literatur nieder. Eines der bekanntesten Beispiele ist der Roman des ivorischen Schriftstellers Amadou Kourouma Les soleils des indépendances (1970; dt. Der Schwarze Fürst, 1980).

An Hand einer Untersuchung über Ousmane Sembènes Roman „Les bouts de bois de Dieu“ (1996; dt. Gottes Holzstücke, 1988) hat die senegalesische Germanistin Khadi Fall die Problematik der Übersetzung frankophoner afrikanischer Literatur in eine andere europäische Sprache behandelt. Dabei brachte sie auf den Punkt, dass es sich dabei um einen Übertragungsvorgang aus zweiter Hand handelt, da der französischen Fassung eine afrikanische Weltansicht des Autors zugrunde liegt. Es gebe aber einen Unterschied zwischen der Übersetzung aus zweiter Hand und der Übersetzung von afrikanischen Werken, die in einer europäischen Sprache geschrieben sind. Dieser Unterschied bestehe darin, dass die Übersetzer von Texten afrikanischer Autoren keine Möglichkeit haben, sich an das Original zu halten, da es in solch einem Fall kein geschriebenes Original gibt, ,weil der Autor eine , innerliche Übersetzung' aus seiner Muttersprache, in der er denkt, in die von ihm benutzte europäische Sprache vorgenommen hat". Daher kritisierte Khadi Fall in ihrer Untersuchung, dass Les bouts de bois de Dieu vom deutschen Übersetzer als Urtext, als das „erste Original“ behandelt wurde. Das führte dazu, dass nicht nur die afrikanische Kultur, sondern auch eine „poetische Andersheit“, die der senegalesische Schriftsteller in seinem Roman aus dem Wolof ins Französische zu übertragen versuchte, in der deutschen Übersetzung nicht angemessen und hinreichend berücksichtigt wurden (vgl. Fall 1996:3f ). ${ }^{7}$

\section{Schlussfolgerung}

In vielen Ländern Subsahara-Afrikas wird Französisch als alleiniges offizielles oder kooffizielles Kommunikationsmittel angewandt. Ungeachtet der Verbreitung eines frankophoniekritischen Diskurses gilt das Französische nach wie vor als Mittel des gesellschaftlichen Aufstiegs in Afrika. Französisch wird vorwiegend von den Eliten der vielsprachigen und multiethnischen Gesellschaften Afrikas benutzt. Die Beziehungen zwischen Französisch und den einheimischen Sprachen verzeichnet einen erfreulichen Umstand, eine positive Entwicklung:

Dass die Francophonie durchaus lernfähig ist, zeigt sich nicht zuletzt daran, wie sie von der lange Zeit auf Assimilation setzenden Politik französischer Einsprachigkeit abrückte und nun stärker auf die Förderung und den Erhalt der sprachlichen und kulturellen Vielfalt setzt. [...]. 
Zudem gewinnt unter Bildungsexperten die Einsicht an Verbreitung, dass in Afrika bessere Bildung und bessere Französischkenntnisse erreicht würden, wenn zumindest in den ersten Schuljahren systematisch in den Herkunftssprachen unterrichtet würde.

Dies schafft Raum für einen Anfang und für eine Vision, jene vom Erhalt und von der Förderung der kulturellen Vielfalt in Afrika. (Erfurt 2005:189)

Die vorliegende Bestandaufnahme bietet sich als Grundlage einer weiteren grundlegenden Überlegung über die Zukunft der französischen Sprache in einem mehrsprachigen und multiethnischen Afrika an. Hoffentlich wird diese Entwicklung der Grundphilosophie des senegalesischen Gelehrten und Vorreiters der Frankophonie-Bewegung Léopold Sédar Senghor (1906-2001) gerecht: Sich selbst bleiben, ohne uns dem Anderen zu verschließen.

\section{Anmerkungen}

1. Das Nouchi ist eine hybride Varietät des Französischen, ,deren syntaktische Muster aus dem Französischen stammen, während im Wortschatz und in der Morphologie, insbesondere in der Verbmorphologie, auf Muster aus ivorischen Sprachen und manchmal auch aus europäischen Sprachen zurückgegriffen wird“ (Erfurt 2005:177.)

2. „Die französische Sprache ist für uns ein Kommunikationsmedium zwischen unseren Staaten und den verschiedenen ethnischen Gruppen innerhalb desselben Staates; sie ist auch eine, die uns den Anschluss an den Rest der Welt ermöglicht. Durch ihre Feinheiten und die Kraft der Schrift hat sie im Laufe der Jahrhunderte bestimmte Werte entwickelt; diese Werte können einen glücklichen Einfluss auf die moderne Entwicklung unserer eigenen Sprachen ausüben, die inzwischen den Anforderungen der schriftlichen Kultur ausgesetzt sind. Unsere Sprachen haben auch ihre Werte, deren Grundlage die Oralität ist; sie sind also in der Lage, die französische Sprache dynamisch zu machen und sie durch ihre Nuancen zu färben. Davon zeugen unsere Meisterwerke, zum Beispiel diejenigen von Léopold Sédar Senghor und Aimé Césaire." (Übersetzung durch L.Y.)

3. „Die sich heutzutage in unseren Leben verkörpert hat und unsere kulturelle Identität vermitteln kann sowie uns ermöglichen, zu erkennen, was wir mit den anderen Völkern der Welt gemeinsam haben." (Mendo Ze, zitiert nach Erfurt 2005:51)

4. „Die Negro-Afrikaner müssen nicht einfach eine Sprache erleiden, die ihnen total fremd ist. Sie müssen nicht nur schlechte Konsumenten der französischen Sprache sein; die Afrikaner müssen die französische Sprache neu schaffen, damit sie ihrem Lebensstil und ihrer Denkweise gerecht wird.“ (Übersetzung durch L.Y.)

5. „Der Ruhm des Verräters“ (Übersetzung durch L.Y.).

6. Vgl. Fall 1996, Fußnote 25. Sie bezieht sich auf eine Untersuchung von Jürgen v. Stackelberg (Klassische Autoren des schwarzen Erdteils, München 1981)

7. Vgl. dazu Traoré 1992:429-439.

\section{Bibliographie}

Erfurt, J. 2005. Frankophonie. Sprache, Diskurs, Politik. Tübingen, Basel: A. Francke Verlag. Fall, K. 1993. Das 'erste' und das 'zweite' Original. Welche Bedingungen muss man bei der Übersetzung negro-afrikanischer frankophoner Literatur betrachten? In B. Thum und G. L. Fink (Hgg.) Praxis interkultureller Germanistik. München: Iudicium. pp. 967-974.

Fall, K. 1996. Ousmane Sembènes Roman "Les bouts de bois de Dieu": Ungeschriebener Wolof-Text, französische Fassung, deutsche Übersetzung. Frankfurt a. M.: IKO. 
Gassama, M. 1995. La langue d'Ahmadou Kourouma ou le français sous le soleil d'Afrique. Paris: Karthala et ACCT.

Kirschbaum, I.v. 1995. Das Hilfsprogramm der UNESCO für den Aufbau staatlicher Schulsysteme in Tropisch-Afrika von 1960 bis 1968 : mit 16 Tabellen. Köln, Univ., Diss.

Kita, J.K. 2008. Afrikanische und europäische Mentalitäten im Vergleich. Mit Beispielen aus der Beratungsarbeit. 2., aktualisierte Auflage, Berlin: Lit Verlag.

Kourouma, A. 1970. Les soleils des indépendances. Paris: Editions du Seuil . (dt. 1980. Der Schwarze Fürst. Wuppertal: Peter Hammer Verlag).

Kubé, S. 2005. La Francophonie vécue en Côte d'Ivoire. Paris: L'Harmattan.

Makouta-Mboukou, J-P. 1973: Le français en Afrique noire (Histoire et méthodes de l'enseignement du français en Afrique noire). Paris: Bordas.

Mendo Ze, G. 1999. Le Français langue africaine. Enjeux et atouts pour la francophonie. Paris: Publisud.

Stackelberg, J. v. 1984. Übersetzungen aus zweiter Hand. Rezeptionsvorgänge in der europäischen Literatur vom 14. Bis zum 18. Jahrhundert. Berlin, New York: De Gruyter.

Tene, A. N. 2004. (Bi)kulturelle Texte und ihre Übersetzung. Romane afrikanischer Schriftsteller in französischer Sprache und die Problematik ihrer Übersetzung ins Deutsche. Würzburg: Königshausen \& Neumann.

Traoré, K. 1992. Kulturelle Dimension der literarischen Übersetzung: Die deutsche Fassung von Kouroumas „Les soleils des indépendances“. Französisch Heute: 429-439.

Yéo, L. 2003. Fremdsprachen als Muttersprachen in Afrika. Chance oder Schande? Überlegungen zum Gebrauch von europäischen Idiomen als Verkehrs- Literatur-, Unterrichts- und Amtssprachen im postkolonialen Afrika. Muttersprache. Vierteljahresschrift für deutsche Sprache 4: 328-340.

\section{Biographical note}

Dr. Lacina Yéo was born in Ferkessedougou in the northern part of the Ivory Coast in 1970. After completing his Master's thesis in 1996 at the Université de Cocody-Abidjan, he received his Ph.D. in 2003 in German languages and literature studies at the Freie Universität Berlin and the Universite de Paris IV-Sorbonne for his work titled "The rehabilitation of Black Africa in the most recent German literature - presented against the background of postcolonial discourse". Since 2003/2004 he has been lecturing in the field of German language and literature as well as applied geography at the Université de Cocody-Abidjan and in the field of intercultural pedagogics at the Freie Universität Berlin. Currently he is working on his postdoctoral lecture thesis with the title "Léopold Sédar Senghor and Janheinz Jahn as literature- and cultural scientists between Africa and the German-speaking world". 